\title{
Partial Face Recognition: An Alignment Free Approach
}

\author{
Shengcai Liao and Anil K. Jain* \\ Department of Computer Science and Engineering \\ Michigan State University, East Lansing, MI 48824, U.S.A. \\ $\{$ scliao, jain\}@cse.msu.edu
}

\begin{abstract}
Many approaches have been developed for holistic face recognition with impressive performance. However, few studies have addressed the question of how to recognize an arbitrary image patch of a holistic face. In this paper we address this problem of partial face recognition. Partial faces frequently appear in unconstrained image capture environments, particularly when faces are captured by surveillance cameras or handheld devices (e.g. mobile phones). The proposed approach adopts a variable-size description which represents each face with a set of keypoint descriptors. In this way, we argue that a probe face image, holistic or partial, can be sparsely represented by a large dictionary of gallery descriptors. The proposed method is alignment free and we address large-scale face recognition problems by a fast filtering strategy. Experimental results on three public domain face databases (FRGCV2.0, AR, and LFW) show that the proposed method achieves promising results in recognizing both holistic and partial faces.
\end{abstract}

\section{Introduction}

Face recognition deals with verifying or identifying a face from its image. It has received substantial attention and its performance has advanced significantly over the last three decades due to its value both in understanding how the face recognition process works in humans as well as in addressing many applications, including access control and video surveillance. While face recognition in controlled conditions (frontal face and uniform illumination) has already achieved satisfactory performance, there still exist many challenges in uncontrolled scenarios, such as nonfrontal pose, facial occlusion, and illumination variations.

Typical applications of face recognition in uncontrolled environments include recognition of individuals in video surveillance frames and images captured by handheld devices (e.g. a mobile phone), where a face may be captured

\footnotetext{
*Anil K. Jain is also with the Dept. of Brain and Cognitive Engineering, Korea University, Anam-dong, Seongbuk-gu, Seoul 136-713, Korea.
}

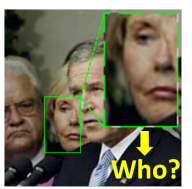

(a)

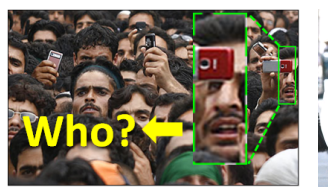

(b)

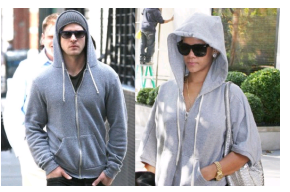

(c)
Figure 1. Examples of partial faces. (a) Partial faces from the LFW database [15]. (b) Many occluded faces in a crowd ${ }^{1}$. (c) Two persons who are trying to hide their identities by wearing hooded sweatshirt and sunglasses ${ }^{2}$.

Table 1. Categories of partial faces

\begin{tabular}{|l|l|}
\hline Category & Example \\
\hline External occlusion & occlusion by other objects \\
\hline Self occlusion & non-frontal pose \\
\hline Facial accessories & hat, sunglasses, scarf, mask \\
\hline Limited field of view & not completely in camera's FOV \\
\hline Extreme illumination & gloomy or highlighted facial area \\
\hline Sensor saturation & underexposure or overexposure \\
\hline
\end{tabular}

in arbitrary pose without user cooperation. In such scenarios, it is quite likely that the captured face image is not a holistic face. These scenarios raise the following important questions: (i) is it possible to recognize a person from a partial image of his face? and (ii) which portion of the face and what size of the partial face are critical for accurate recognition? We call the resulting problem as Partial Face Recognition (PFR), so as to differentiate it from the holistic face recognition problem. State-of-the-art face recognition systems [1, 2] have difficulties with the general PFR problem.

Partial face images result due to many factors, which are categorized in Table 1. Fig. 1(a) shows some examples of partial faces which include a non-frontal face, a face occluded by another face, and a face that is not completely visible in the image frame. Images or videos captured in unconstrained environments often contain partial faces that are difficult to recognize by state-of-the-art face recognition

\footnotetext{
${ }^{1}$ http://www.textually.org/picturephoning/archives/2008/09/021247.htm

${ }^{2}$ http://www.howtovanish.com/2010/01/avoid-nosy-surveillancecameras/
} 
systems. Therefore, research on partial face recognition is important to advance the state of the art. As an example, a PFR system will enable law enforcement agencies to identify a suspect in a crowd from his partial face captured by a mobile phone and match it to a watch list through a wireless link. Figs. 1(b) and (c) show two scenarios in uncontrolled environments, where (b) many occluded faces exist in a crowd; and (c) two persons are trying to hide their identities by wearing hooded sweatshirt and sunglasses.

To our knowledge, almost all of the current face recognition systems require face alignment. To align a face, the most popular approach is to first detect the two eyes and then normalize the face image geometrically. However, this approach will fail as long as one eye is invisible in a partial face image. Other face alignment methods include Active Shape Model (ASM) [9] and Active Appearance Model (AAM) [8]. They depend on localization of a certain fixed number of landmarks in the holistic face image.

Among various types of partial faces, in practice the occluded and non-frontal ones are the most frequently encountered. While several papers have dealt with occlusion $[17,24,14,16]$, they still require well aligned face images to compensate for the occlusion effect. Ekenel and Stiefelhagen showed that face alignment plays a key role in recognition performance in case of occlusion [10].

Wright et al. introduced sparse representation classification (SRC) scheme for face recognition [34], which was further improved in [32] to compensate for some possible registration errors. Good performance under small amounts of pose and occlusion variations was reported. However, S$\mathrm{RC}$ is not applicable to partial face images with large pose variations or limited field of view (see Fig. 1(a)).

Non-frontal face recognition has also attracted significant attention, including multi-view [4, 26] and cross-view face recognition $[30,11,13,33]$. A critical step in these approaches is to localize a certain fixed number of representative facial landmarks and establish landmark correspondences between the input image and the target image in different views. As a result, both images are required to have visible anchor points. This requirement would not be satisfied in case of external occlusion or limited field of view.

Yi et al. developed a recognition method [35] for frontal, near infrared images with limited field of view. While this method achieved promising matching accuracy, it requires high resolution images with good skin texture, and it has difficulty with pose variations.

Some research on partial face recognition that only require face sub-images as input has been reported. Sato et al. [29] showed that certain facial sub-images (such as eye, nose, and ear) could be used for recognition. Gutta et al. [12] showed that a half (left or right) of the face is sufficient for the recognition task. Park et al. showed that the periocular region is useful for identification [25]. Nevertheless, these approaches require the presence of expected and predefined facial components in the face image for recognition.

Instead of holistic representation, some face recognition approaches adopted part-based fusion to deal with occlusion and pose variation $[6,26,13,22]$. These approaches either manually divide the face image into several subregions, or automatically detect several predefined components (e.g. eye, nose, and mouth), and fuse results from individual parts for recognition. However, their performance deteriorates when some of the facial portions are invisible and cannot be localized.

For images that are difficult to align (e.g. flowers), bagof-words (BoW) representation is frequently used for visual object categorization, which applies clustering techniques to build "visual words". However, histogram of visual words of a partial face may be quite different from that of the whole face. In [20], the BoW representation was applied for face recognition, but it required pre-alignment so that face images could be divided into regular blocks.

In this paper, we present a general formulation of the partial face recognition problem. We do not know a priori whether the input face is holistic or partial. Further, we do not assume the presence of the eyes or any other facial component in the image, and we do not assume any prior information about face alignment. We provide a general matching solution to all types of partial faces listed in Table 1. Our goal is to recognize an arbitrary partial face without alignment. This is achieved by first deriving a multi keypoint descriptor based representation and constructing a gallery dictionary accordingly. Then multi-task sparse representation is learned from features of each probe face, and recognition is done by the returned sparse coefficients. We call the proposed method as multi keypoint descriptor based sparse representation classification (MKD-SRC).

The various approaches we have reviewed along with the proposed one are summarized in Table 2. The novelties of the proposed approach are: (i) it addresses the general partial face recognition problem without an alignment stage; (ii) by applying multi keypoint descriptors in SRC, it is a unified face recognition framework for both holistic and partial faces; (iii) a fast filtering strategy is proposed to address large-scale face recognition.

\section{Proposed Approach}

\subsection{Gallery Dictionary Construction}

To allow for general partial face recognition capability, we construct a large gallery dictionary of multi keypoint descriptors (MKD) as follows. First, we detect multiple SIFT keypoints [21] in each image, and compute the corresponding descriptors. For each class (subject) $c$ in the gallery, suppose $k_{c}$ keypoints, say, $\mathbf{p}_{c_{1}}, \mathbf{p}_{c_{2}}, \cdots, \mathbf{p}_{c_{k_{c}}}$, are detected. Note that if class $\mathrm{c}$ has multiple images, we sim- 
Table 2. A comparison of partial face recognition approaches along with the proposed one

\begin{tabular}{|c|c|c|c|}
\hline Problem addressed & Approach & Face image requirement & Gallery size \\
\hline \multirow{3}{*}{ Occlusion } & Subspace [17, 24] & & \\
& SRC [34, 32] & & \\
& SVM [14, 16] & Frontal, aligned and cropped & $\leq 1,196$ \\
& $\begin{array}{c}\text { Part-based fusion [6, 26, 13, 22] } \\
\text { Single component [29, 12, 25] }\end{array}$ & & $\leq 250$ \\
\hline Pose & Multi-view [4, 26] & Alignment via landmarks & $\leq$ \\
\hline Cross-view [30, 11, 13, 33] & Skin texture [35] & Frontal, partial face alignment & 114 \\
\hline Occlusion, pose, limited FOV & MKD-SRC (proposed) & Alignment free & $>10,000$ \\
\hline
\end{tabular}

ply pool the keypoints extracted from all the images from class c. The corresponding $k_{c}$ SIFT descriptors are denoted as $\mathbf{d}_{c_{1}}, \mathbf{d}_{c_{2}}, \cdots, \mathbf{d}_{c_{k_{c}}}$, where each descriptor is an $M$ dimensional vector (in our case, $M=128$ ). Let

$$
\mathbf{D}_{c}=\left(\mathbf{d}_{c_{1}}, \mathbf{d}_{c_{2}}, \cdots, \mathbf{d}_{c_{k_{c}}}\right) \text {. }
$$

Then the descriptors from the same class form a subdictionary of size $M \times k_{c}$ representing class $c$. A gallery dictionary for all the $C$ classes is built as

$$
\mathbf{D}=\left(\mathbf{D}_{1}, \mathbf{D}_{2}, \cdots, \mathbf{D}_{C}\right)
$$

Note that $\mathbf{D}$ has a total number of $K=\sum_{c=1}^{C} k_{c}$ descriptors. Hence the dictionary size is $M \times K$.

Inspired by [34], we adopt a sparse representation scheme for recognition, and express any descriptor from a probe image in terms of a sparse linear combination of the gallery dictionary $\mathbf{D}$.

\subsection{Multi-Task Sparse Representation}

Let us consider a probe face image with $k$ descriptors

$$
\mathbf{Y}=\left(\mathbf{y}_{1}, \mathbf{y}_{2}, \cdots, \mathbf{y}_{k}\right)
$$

We aim to solve a multi-task $l_{1}$-minimization problem

$$
\hat{\mathbf{X}}=\arg \min _{\mathbf{X}} \sum_{i=1}^{k}\left\|\mathbf{x}_{i}\right\|_{1}, \text { s.t. } \mathbf{Y}=\mathbf{D X}
$$

where $\mathbf{X}=\left(\mathbf{x}_{1}, \mathbf{x}_{2}, \cdots, \mathbf{x}_{k}\right) \in R^{K \times k}$ is the sparse coefficient matrix, and $\|\cdot\|_{1}$ denotes the $l_{1}$ norm of a vector. This is equivalent to solving the following $k l_{1}$-minimization problems for each probe descriptor $y_{i}$

$$
\hat{\mathbf{x}}_{i}=\arg \min _{\mathbf{x}_{i}}\left\|\mathbf{x}_{i}\right\|_{1}, \text { s.t. } \mathbf{y}_{i}=\mathbf{D} \mathbf{x}_{i}, \quad i=1,2, \cdots, k .
$$

We adopt the following multi-task sparse representation based classification to determine the identity of the probe image, which is inspired from [34]

$$
\min _{c} r_{c}(\mathbf{Y})=\frac{1}{k} \sum_{i=1}^{k}\left\|\mathbf{y}_{i}-\mathbf{D}_{c} \delta_{c}\left(\hat{\mathbf{x}}_{i}\right)\right\|_{2}^{2},
$$

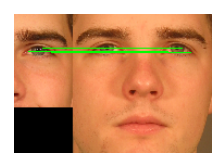

(a) SIFT (Genuine)

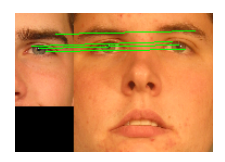

(b) SIFT (Impostor)

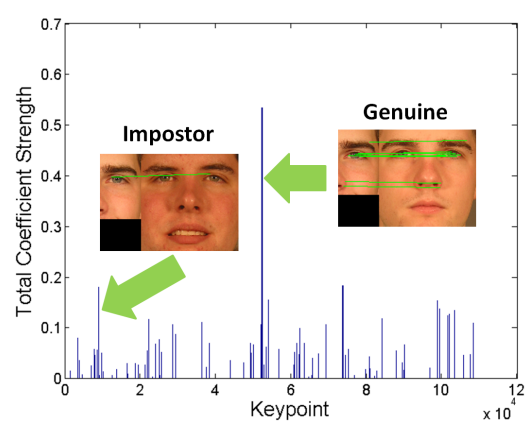

(c) MKD-SRC
Figure 2. Matching examples with a partial face image as probe. (a) SIFT matching: 3 keypoints are matched for a genuine pair. (b) SIFT matching: 5 keypoints are matched for an impostor pair. (c) MKD-SRC coefficient distribution and the corresponding matches. Horizontal axis: keypoints correspond to columns of $\mathbf{D}$; vertical axis: coefficient strength, as computed by $\sum_{j=1}^{k}\left|\hat{x}_{i j}\right|, i=1$, $2, \cdots, K$, where $k=15$ in this example. The maximum coefficien$\mathrm{t}$ strength corresponds to a genuine match (right subplot), where 8 correspondences are drawn because 8 probe descriptors get the maximum coefficients at gallery keypoints of this user. In contrast, the left subplot corresponds to an impostor match.

where $\delta_{c}(\cdot)$ is a function which selects only the coefficients corresponding to class $c$. We call the resulting algorithm as multi keypoint descriptor based SRC (MKD-SRC). Note that MKD-SRC does not need an alignment stage.

Fig. 2(c) illustrates the MKD-SRC approach, where the solution is indeed sparse with zero or near zero coefficients at many keypoints, and the probe partial face is correctly recognized. In contrast, the direct SIFT matching approach [21] misclassifies the same probe image (Figs. 2(a) \& (b)). This example shows that MKD-SRC successfully exploits the gallery class information and suppresses matches between impostor pairs by seeking the sparsest representation among all the gallery images.

\subsection{Fast Filtering}

Taking into account the very large value of $K$ (in practice, $K$ can be of the order of millions) in the dictionary 
D, solving Eq. (5) will be computationally challenging in practice. Therefore, we adopt a fast approximate solution. For each probe descriptor $\mathbf{y}_{i}$, we first compute the following linear correlation coefficients between $\mathbf{y}_{i}$ and all the descriptors in the dictionary $\mathbf{D}$

$$
\mathbf{c}_{i}=\mathbf{D}^{T} \mathbf{y}_{i}, i=1,2, \cdots, k .
$$

Then for each $\mathbf{y}_{i}$, we filter out only $L(L \ll K)$ descriptors according to the top $L$ largest values in $\mathbf{c}_{i}$, resulting in a small sub-dictionary $\mathbf{D}_{M \times L}^{(i)}$. Next, $\mathbf{D}$ is replaced by $\mathbf{D}^{(i)}$ in Eq. (5), and Eq. (6) is adjusted accordingly.

As we will see later, this approximate solution is much faster, with no significant degradation in performance. From Eq. (7) and the selection of top $L$ elements (note that this can be done in $O\left(K \log _{2} L\right)$ by the partial quick sort algorithm) we know that the computation time of the filtering step scales linearly with respect to $K$ (the number of gallery keypoints) and $k$ (the number of probe keypoints), and hence scales almost linearly with respect to the gallery size for each probe image (considering an average number of keypoints per image).

\subsection{Differences with Related Methods}

The differences between the proposed method and three related methods for face recognition are listed in Table 3. Both SIFT [21] and MKD-SRC use variable-size description; they are alignment free and can be used for both holistic and partial face recognition. In contrast, both SRC [34] and LBP-SRC [7] (which uses LBP histograms instead of pixel values in SRC) use a fixed-length description; they require face alignment and can not be applied to general partial face recognition problem. All SRC based method$\mathrm{s}$ exploit gallery class information for face recognition, but SIFT matches each pair of images separately.

The difference among SRC, LBP-SRC and MKD-SRC lies in the feature representation. Since both SRC and LBPSRC require face alignment and use a single fixed-size feature vector to represent an image, each column of their corresponding dictionaries is related to one gallery image. However, in such a scheme a partial face might have difficulty in alignment and representation due to some unknown missing facial regions. In contrast, MKD-SRC uses a variable-size description; each image is represented by a set of descriptors. The MKD dictionary is composed of a large number of gallery descriptors, making it possible to sparsely represent descriptors from a probe face, regardless of being holistic or partial. Note that although LBP-SRC also extracts local features (LBP histograms) from the image, they are extracted at a certain fixed number of predefined locations after alignment.

Some other existing face recognition approaches based on SIFT include [5, 18, 20, 19], however, all of them depend on pre-aligned face images.
Table 4. Databases used in our experiments

\begin{tabular}{|l|c|c|c|}
\hline Database & FRGCv2.0 & AR & LFW \\
\hline Scenario & partial patch & occlusion & pose \& occlusion \\
\hline \#Gallery & 11,398 & 1,331 & 20,489 \\
\hline \#Probe & 14,630 & 1,530 & 2,744 \\
\hline \#Subjects & 10,466 & 1,331 & 15,749 \\
\hline
\end{tabular}

\section{Experiments}

In this section, we provide experimental results on three public domain databases: FRGCv2.0, AR, and LFW, which are summarized in Table 4. With these three databases we focus on three scenarios of partial face recognition: arbitrary patch of holistic face, occluded face, and face images with arbitrary occlusion and pose variations, respectively. Since in surveillance scenarios, identification is a more reasonable mode than verification, we performed identification experiments on these three databases and used the Cumulative Matching Characteristic (CMC) curve for performance evaluation. We compared the proposed MKD-SRC method to the SIFT matching approach [21], as well as a commercial face recognition software FaceVACS [1] and the original SRC algorithm [34] if they are applicable.

\subsection{Partial Face Recognition with Arbitrary Patch}

\subsubsection{Database}

To evaluate the proposed method, we synthetically generated a large database of partial faces. We used 16,028 frontal face images of 466 subjects from the Face Recognition Grand Challenge Ver2.0 (FRGCv2.0) database [28]. The selected images were randomly divided into gallery and probe subset. The gallery set consisted of 1,398 images of 466 subjects, with 3 images per subject. The remaining 14,630 images composed the probe set. To make the identification problem more challenging, we included an additional 10,000 frontal face images ( 1 image per subject) from a private database to enlarge the gallery set. Therefore, the gallery set contained 11,398 images in total.

All gallery face images were cropped to $128 \times 128$ pixels according to the two eye coordinates provided. Fig. 3(a) shows some examples of cropped face images. The probe images were first cropped in the same manner as the gallery images. Next, for each cropped probe image, a patch at random position of a random size $h \times w$ was cropped to represent a partial face, where both $h$ and $w$ were uniformly distributed in $[50,100]$. Fig. 3(b) shows some instances of such randomly cropped face patches. Our objective is to recognize these arbitrary patches (we assume they have been manually cropped, as commonly made in forensic applications), which can be viewed as partial faces with external occlusions or limited FOV. Note that the position information of how these patches were cropped was not known 
Table 3. Differences between the proposed and three related face recognition algorithms

\begin{tabular}{|c|c|c|c|c|}
\hline & SIFT [21] & SRC [34] & LBP-SRC [7] & MKD-SRC \\
\hline Size of descriptor per image & variable & fixed & fixed & variable \\
\hline Face image requirement & alignment free & aligned and cropped & aligned and cropped & alignment free \\
\hline Gallery class information & not used & utilized & utilized & utilized \\
\hline Holistic face & yes & yes & yes & yes \\
\hline Arbitrary partial face & yes & no & no & yes \\
\hline
\end{tabular}

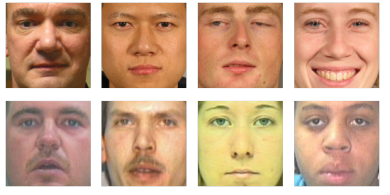

(a) gallery

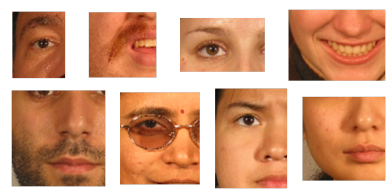

(b) probe
Figure 3. Example face images: (a) gallery images from the FRGCv2.0 database (upper row) and the extended gallery set (bottom row); (b) partial face images cropped from FRGCv2.0.

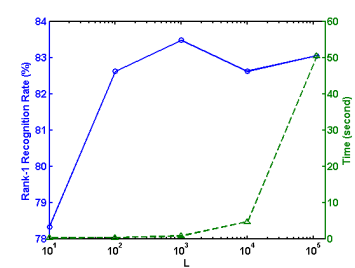

\begin{tabular}{|l|l|l|}
\hline L & Accuracy & Time \\
\hline $10^{1}$ & $78.33 \%$ & $0.2 \mathrm{~s}$ \\
\hline $10^{2}$ & $82.62 \%$ & $0.3 \mathrm{~s}$ \\
\hline $10^{3}$ & $83.48 \%$ & $0.8 \mathrm{~s}$ \\
\hline $10^{4}$ & $82.62 \%$ & $4.7 \mathrm{~s}$ \\
\hline$=K$ & $83.05 \%$ & $50.4 \mathrm{~s}$ \\
\hline
\end{tabular}

Figure 4. Performance in both speed (average computation time per probe for a gallery of 1,398 images) and rank-1 accuracy, with respect to the parameter $L$, on the FRGCv2.0 database.

to any of the recognition algorithm used in our evaluation.

Next we built an MKD based dictionary from the gallery images, and also extracted multi keypoint descriptors for each probe image. The MKD dictionary was of size $128 \times$ $1,181,514$, while the number of descriptors for all probe images was 280, 838. To solve Eq. (5), we utilized the L1 Homotopy MATLAB toolbox [3].

\subsubsection{Parameter Selection and Computation Time}

Our MATLAB implementation of MKD-SRC runs on a PC server with Intel Core i7 $2.93 \mathrm{GHz}$ CPU and 16GB memory. There is one parameter $L$ in the proposed algorithm, as described in Section 2.3. We tried different values of $L$ and evaluated the resulting performance in both speed and accuracy, as shown in Fig. 4. We used a subset of the selected data, including 1,398 gallery images and 466 probe images of the 466 subjects. This resulted in $K=111,643$ for the dictionary. Using all the $K$ gallery descriptors (Eq. (7) was not computed), the rank-1 recognition rate is $83.05 \%$, with an average recognition time of 50.4 seconds per probe. From Fig. 4 we can see that $L=100$ and $L=1000$ have a good balance between computation time and accuracy. We decided to set $L=100$ for all the following experiments.

Next we evaluated MKD-SRC on the large-scale dataset

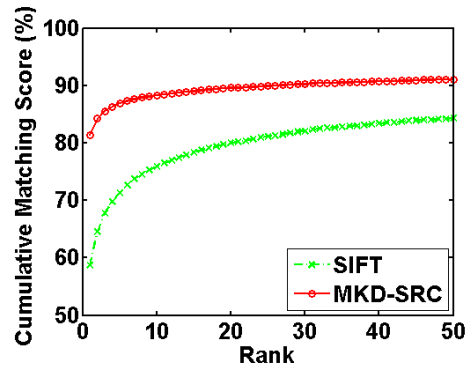

Figure 5. CMC curves on face patches extracted from the FRGCv2.0 database.

(11,398 gallery images and 14,630 probe images). The average number of keypoints per gallery image and per probe image were 104 and 19, respectively. A complete solution of the MKD-SRC framework consists of three steps: keypoint detection, gallery keypoint filtering (by Eq. (7)), and multi-task sparse representation and classification. For a probe image, the average computation times for the first and the last steps are 0.07 and 0.61 seconds, respectively, which are independent of the gallery size. For the second step, it takes 0.80 seconds per probe, on average. As analyzed in Section 2.3, the computation time of the second step scales approximately linearly with respect to the gallery size. In summary, the whole matching process takes 1.48 seconds per probe for a gallery of 11,398 images.

\subsubsection{Performance and Comparison}

The proposed MKD-SRC algorithm was compared to the SIFT matching approach with the same keypoints and descriptors [21]. The SRC algorithm was not compared because it is not applicable to partial face recognition without prior alignment. Fig. 5 shows the performance of the cumulative matching characteristic (CMC) curves of the two algorithms. It can be seen that the proposed MKD-SRC algorithm significantly improves partial face recognition performance compared to that of the SIFT matching. The rank1 recognition rate of MKD-SRC is $81.31 \%$, while that of SIFT matching is $58.70 \%$.

Figs. 6 and 7 show examples of some correctly and incorrectly recognized partial faces, respectively, from the FRGCv2.0 database. It appears that the periocular region contains rich information for recognition, while face patch- 

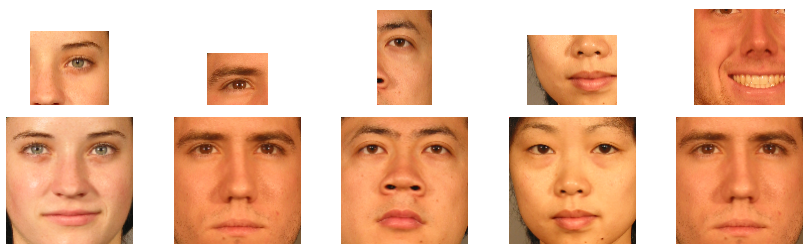

Figure 6. Correctly recognized partial faces (first row) synthesized from the FRGCv2.0 database and the corresponding gallery images (second row).
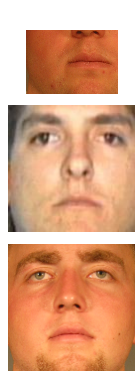
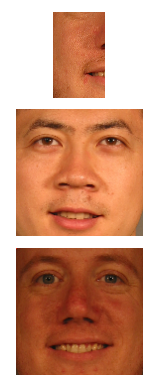
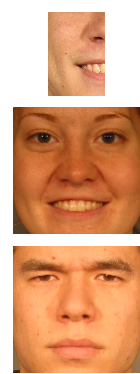
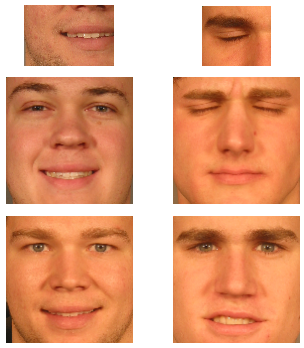

Figure 7. Misclassified partial faces (top row) cropped from the FRGCv2.0 database, and the corresponding true gallery images (bottom row) and the false matches at rank-1 (middle row).

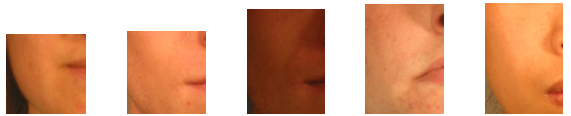

Figure 8. Partial face images with no keypoints detected.

es with closed eyes, or partial mouth/cheek are not easy to recognize (note that only three images per subject were randomly selected in the gallery set). For a partial mouth patch or a cheek patch, very few keypoints could be detected. Fig. 8 shows some probe images where no keypoints could be detected.

\subsection{Holistic Face Recognition with occlusion}

For a more convincing experimental evaluation and comparison, we performed two additional experiments on holistic faces with staged occlusions and real occlusions. The first experiment was done on the AR database [23] with staged occlusions. The AR database contains 135 subject$\mathrm{s}$ with 76 males and 59 females. We selected one image (neutral expression) per class as the gallery set, and 1,530 images, all with sunglasses or scarf, as the probe set. Each probe image may have left-side or right-side illumination. We added 1,196 frontal images from the FERET database [27] to increase the gallery size. All images were cropped to $128 \times 128$ pixels after face detection. No alignment was done between the probe and gallery sets. Fig. 9 shows some cropped face images from the AR database. It can be seen that they are not aligned very well.

We compared the proposed method to SIFT matching [21], SRC [34], and FaceVACS [1]. The alignment stage was used only in FaceVACS. Fig. 10 shows the CMC
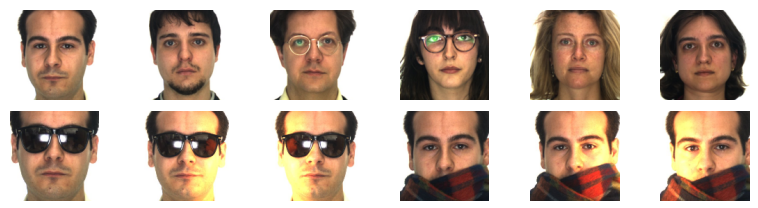

Figure 9. Typical face images from the AR database. Top row: gallery images. Bottom row: probe images.

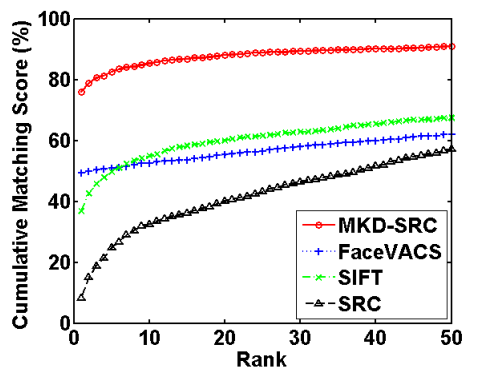

Figure 10. CMC curves on the AR database.

curves of these methods on the AR database. These results indicate that MKD-SRC outperforms other algorithms with a notable margin, achieving rank-1 recognition rate of $76.01 \%$. As expected, FaceVACS does not perform very well (49.41\% rank-1 accuracy) on these occluded probe images. The rank-1 recognition rate for SIFT and SRC are $36.86 \%$ and $8.30 \%$, respectively. Note that this experimen$t$ is really challenging because all the probe images have faces with sunglasses or scarf and also contain illumination variations; meanwhile, only one sample per class with neutral expression is available in the gallery set. While Wright et al. demonstrated that their SRC algorithm showed promising performance on well aligned AR faces with sunglasses and scarf, and with sufficient number of training samples [34], our experiments show that the performance of SRC is not good on unaligned faces with only one training sample per class.

\subsection{Face Recognition on LFW}

While the above experiments were done on synthesized or studio-controlled databases, we performed another experiment on more realistic face images captured in uncontrolled environments: Labeled Faces in the Wild (LFW) [15]. The LFW database was created in an effort to challenge face recognition algorithms in real scenarios. All images in this database were downloaded from the internet. The only condition for including an image in this database was that the face can be detected by the Viola-Jones face detector [31]. The LFW database includes 13,233 images of 5,749 subjects. Face images from LFW contain large variations in pose and illumination, and they might be arbitrarily occluded. Fig. 11 shows some examples from this database.

For our experiments on the LFW database, we select- 


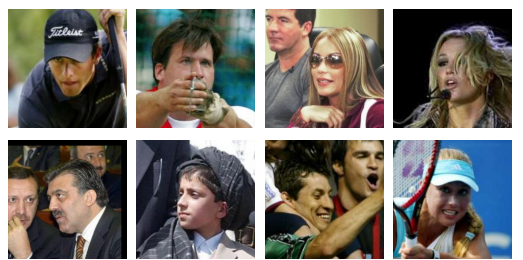

Figure 11. Sample images from the LFW database.

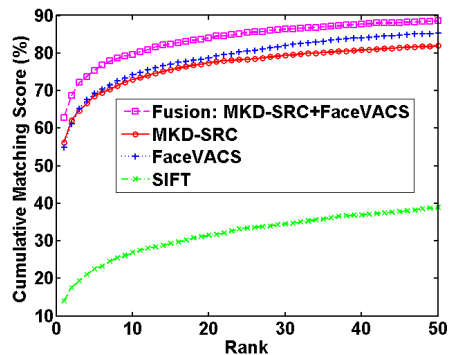

Figure 12. CMC curves on the LFW database.

ed all the 5,749 subjects with up to 10 images per subject to compose the gallery set, which included 10,489 images. The remaining 2,744 images were selected as the probe set. We also added an additional 10,000 background images to enlarge the gallery set. Note that the original LFW bench mark protocol is for 1-vs-1 verification mode. Here, we conducted an identification experiment to simulate the surveillance scenario. Further, we used unaligned versions of LFW images in our experiments. These images contain extended windows (a ratio of 2.2) output by the Viola-Jones face detector, and re-scaled to $250 \times 250$ pixels. We used the face detector output (the center part of $114 \times 114$ pixels) directly for our experiments.

The CMC curves of three face recognition algorithms are depicted in Fig. 12. One can observe that the performance of the proposed MKD-SRC algorithm is similar to that of the state-of-the-art algorithm FaceVACS, and both of them achieve higher recognition rates than SIFT. The rank-1 recognition rates for MKD-SRC, FaceVACS, and SIFT are $56.23 \%, 54.96 \%$, and $13.99 \%$, respectively. Notice that although the recognition rates of both MKD-SRC and FaceVACS are not very satisfactory for this challenging database, it is promising that MKD-SRC could also deal with non-frontal faces. A sum score fusion of MKD-SRC and FaceVACS, also depicted in Fig. 12, shows an improved rank-1 accuracy of $62.79 \%$. Fig. 13 shows examples of probe images that are correctly and incorrectly recognized by MKD-SRC. While MKD-SRC is able to recognize some non-frontal, occluded, or incorrectly localized faces, there still exist many challenging examples that are hard to recognize.

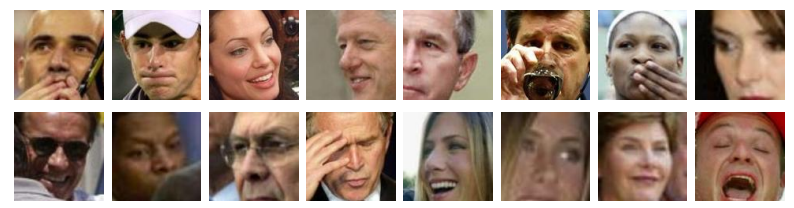

Figure 13. Correctly (top row) and incorrectly (bottom row) recognized face images from the LFW database using the proposed method.

\section{Summary and Future Work}

We have addressed the problem of recognizing a face from its partial image. We have proposed a novel alignment free approach, called MKD-SRC to deal with this problem in general. MKD-SRC represents each face image with a set of keypoint descriptors, and constructs a large dictionary from all the gallery descriptors. In this way descriptors of a probe image can be sparsely represented by the dictionary and the identity of the probe face image can be inferred accordingly. We have shown promising results on synthesized partial faces (from the FRGCv2.0 database), occluded holistic faces (in the AR database), and arbitrarily occluded or non-frontal faces (in the LFW database). Comparison with state-of-the-art approaches shows that the MKDSRC is well suited for the general partial face recognition problem. Our future work would address to improve the description step for a better handling of illumination variations. Furthermore, due to the general framework of MKDSRC, it may be interesting to also apply MKD-SRC to other image classification areas, such as recognition of biometric modalities other than face, and object categorization.

\section{Acknowledgement}

The authors would like to thank Brendan Klare and Unsang Park for their valuable feedback. Anil K. Jain's research was partially supported by the WCU (World Class University) program funded by the Ministry of Education, Science and Technology through the National Research Foundation of Korea (R31-10008).

\section{References}

[1] FaceVACS Software Developer Kit, Cognitec Systems GmbH, http://www.cognitec-systems.de. 1, 4, 6

[2] PittPatt Face Recognition Web Demo, Pittsburgh Pattern Recognition, Inc. http://demo.pittpatt.com/recognition_demo/. 1

[3] S. Asif. L1 homotopy: A MATLAB toolbox for homotopy algorithms in L1 norm minimization problems. http://users.ece.gatech.edu/ sasif/homotopy/. 5

[4] D. Beymer. Face recognition under varying pose. In IEEE Computer Society Conference on Computer Vision and Pattern Recognition, pages 756-761, June 1994. 2, 3

[5] M. Bicego, A. Lagorio, E. Grosso, and M. Tistarelli. On the use of SIFT features for face authentication. In $C V P R W$ 
'06: Proceedings of the 2006 Conference on Computer Vision and Pattern Recognition Workshop, Washington, DC, USA, 2006. 4

[6] R. Brunelli and T. Poggio. Face recognition: Features versus templates. IEEE Transactions on Pattern Analysis and Machine Intelligence, 15(10):1042-1052, 1993. 2, 3

[7] C. Chan and J. Kittler. Sparse representation of (Multiscale) histograms for face recognition robust to registration and illumination problems. In IEEE International Conference on Image Processing, pages 2441-2444, 2010. 4, 5

[8] T. Cootes, G. Edwards, and C. Taylor. Active appearance models. IEEE Transactions on Pattern Analysis and Machine Intelligence, 23(6):681-685, June 2001. 2

[9] T. F. Cootes, C. J. Taylor, D. Cooper, and J. Graham. Active shape models - their training and application. Computer Vision and Image Understanding, 61(1):38-59, Jan 1995. 2

[10] H. Ekenel and R. Stiefelhagen. Why is facial occlusion a challenging problem? Advances in Biometrics, 2009. 2

[11] R. Gross, I. Matthews, and S. Baker. Fisher light-fields for face recognition across pose and illumination. Pattern Recognition, pages 481-489, 2002. 2, 3

[12] S. Gutta, V. Philomin, and M. Trajkovic. An investigation into the use of partial-faces for face recognition. In Proceedings of International Conference on Automatic Face and Gesture Recognition, pages 33-38, 2002. 2, 3

[13] B. Heisele, P. Ho, J. Wu, and T. Poggio. Face recognition: component-based versus global approaches. Computer Vision and Image Understanding, 91(1-2):6-21, 2003. 2, 3

[14] K. Hotta. Robust face recognition under partial occlusion based on support vector machine with local gaussian summation kernel. Image and Vision Computing, 26(11):14901498, 2008. 2, 3

[15] G. B. Huang, M. Ramesh, T. Berg, and E. Learned-Miller. Labeled faces in the wild: A database for studying face recognition in unconstrained environments. Technical Report 07-49, University of Massachusetts, Amherst, October 2007. 1,6

[16] H. Jia and A. Martínez. Support vector machines in face recognition with occlusions. Proceedings of the IEEE Computer Society Conference on Computer Vision and Pattern Recognition, pages 136-141, June 2009. 2, 3

[17] J. Kim, J. Choi, J. Yi, and M. Turk. Effective representation using ICA for face recognition robust to local distortion and partial occlusion. IEEE Transactions on Pattern Analysis and Machine Intelligence, 27:1977-1981, 2005. 2, 3

[18] D. Kisku, A. Rattani, E. Grosso, and M. Tistarelli. Face identification by SIFT-based complete graph topology. In IEEE Workshop on Automatic Identification Advanced Technologies, pages 63-68, 2007. 4

[19] B. Klare, Z. Li, and A. Jain. Matching forensic sketches to mug shot photos. Pattern Analysis and Machine Intelligence, IEEE Transactions on, 33(3):639 -646, march 2011. 4

[20] Z. Li, J. Imai, and M. Kaneko. Robust face recognition using block-based bag of words. In the 20th International Conference on Pattern Recognition, Aug. 2010. 2, 4

[21] D. G. Lowe. Distinctive image features from scale-invariant keypoints. International Journal of Computer Vision, 60:91110, 2004. 2, 3, 4, 5, 6
[22] A. Martínez. Recognizing imprecisely localized, partially occluded, and expression variant faces from a single sample per class. IEEE Transactions on Pattern Analysis and Machine Intelligence, 24(6):748-763, June 2002. 2, 3

[23] A. Martinez and R. Benavente. The AR face database. Technical report, CVC Technical report, 1998. 6

[24] H. J. Oh, K. M. Lee, and S. U. Lee. Occlusion invariant face recognition using selective local non-negative matrix factorization basis images. Image and Vision Computing, 26(11):1515-1523, 2008. 2, 3

[25] U. Park, A. Ross, and A. Jain. Periocular biometrics in the visible spectrum: A feasibility study. In IEEE 3rd International Conference on Biometrics: Theory, Applications, and Systems, pages 1-6, 2009. 2, 3

[26] A. Pentland, B. Moghaddam, and T. Starner. View-based and modular eigenspaces for face recognition. In Proceedings of the IEEE Conference on Computer Vision and Pattern Recognition, pages 84-91, 1994. 2, 3

[27] P. Phillips, H. Wechsler, J. Huang, and P. Rauss. The FERET database and evaluation procedure for face-recognition algorithms. Image and Vision Computing, 16(5):295-306, 1998. 6

[28] P. J. Phillips, P. J. Flynn, T. Scruggs, K. W. Bowyer, J. Chang, K. Hoffman, J. Marques, J. Min, and W. Worek. "Overview of the face recognition grand challenge". In Proceedings of IEEE Computer Society Conference on Computer Vision and Pattern Recognition, 2005. 4

[29] K. Sato, S. Shah, and J. Aggarwal. Partial face recognition using radial basis function networks. In Proceedings of the Third IEEE International Conference on Automatic Face and Gesture Recognition, pages 288-293, 1998. 2, 3

[30] T. Vetter and T. Poggio. Linear object classes and image synthesis from a single example image. IEEE Transactions on Pattern Analysis and Machine Intelligence, 19(7):733741, 1997. 2, 3

[31] P. Viola and M. Jones. Robust real time object detection. In IEEE ICCV Workshop on Statistical and Computational Theories of Vision, Vancouver, Canada, July 13 2001. 6

[32] A. Wagner, J. Wright, A. Ganesh, Z. Zhou, and Y. Ma. Towards a practical face recognition system: Robust registration and illumination by sparse representation. In IEEE Computer Society Conference on Computer Vision and Pattern Recognition, 2009. 2, 3

[33] L. Wiskott, J.-M. Fellous, N. Krüger, and C. von der Malsburg. Face recognition by elastic bunch graph matching. IEEE Transactions on Pattern Analysis and Machine Intelligence, 19(7):775-779, 1997. 2, 3

[34] J. Wright, A. Y. Yang, A. Ganesh, S. S. Sastry, and Y. Ma. Robust face recognition via sparse representation. IEEE Transactions on Pattern Analysis and Machine Intelligence, 31:210-227, 2009. 2, 3, 4, 5, 6

[35] D. Yi, S. Liao, Z. Lei, J. Sang, and S. Li. Partial face matching between near infrared and visual images in MBGC portal challenge. Advances in Biometrics, pages 733-742, 2009. 2, 3 